\title{
Prescription pattern of fixed dose drug combination in cardiology department in a tertiary care hospital
}

\author{
Pramod Kumar Manjhi ${ }^{1}$, Lalit Mohan ${ }^{1}$, Manish Kumar ${ }^{1}$, Harihar Dikshit ${ }^{1}$, \\ B.P. Singh ${ }^{2}$, Anuj Kumar Pathak ${ }^{1}$, Sanjeev Kumar ${ }^{1}$
}

\begin{abstract}
${ }^{1}$ Department of Pharmacology, ${ }^{2}$ Department of Cardiology, Indira Gandhi Institute of Medical Sciences, Sheikhpura, Patna, Bihar, 800014, India
\end{abstract}

Received: 21 March 2016 Accepted: 27 April 2016

*Correspondence to: Dr. Lalit Mohan, Email: drlalitjee@rediffmail. com

Copyright: () the author(s), publisher and licensee Medip Academy. This is an openaccess article distributed under the terms of the Creative Commons Attribution NonCommercial License, which permits unrestricted noncommercial use, distribution, and reproduction in any medium, provided the original work is properly cited.

\begin{abstract}
Background: A cardiovascular disease (CVD) is one of the most common causes of mortality and morbidity globally. The drastic change in the lifestyle of population during $21^{\text {st }}$ century has had a great impact on health especially cardiovascular diseases. Objective of this study was to assess the prescription pattern of fixed dose drug combinations (FDCs) in the department of cardiology in a tertiary care hospital.

Methods: The prescriptions of 210 patients suffered by cardiovascular disorders who attended the department of cardiology were analysed and following data were collected - patient demographics, diagnosis, dosage form, therapeutic category, cost of FDCs compared to individual drugs, duration of therapy, the total number of drugs per prescription and FDCs prescribed, composition of the FDC, whether FDC prescribed is approved by WHO list of essential medicine. A descriptive analysis of data was done.

Results: 15 types of FDCs prescribed were received by $92(43.80 \%)$ individuals among 210 patients who attended the cardiology OPD. Prescription of FDCs was most common $28(30.43 \%)$ in patients age group 51-60 years. The commonly prescribed FDC was aspirin with atorvastatin followed by telmisartan with hydrochlorothiazide.

Conclusions: The results suggested that FDCs for cardiovascular disorders are frequently prescribed by cardiologist. However all are not approved by WHO list of essential medicine. This study was focused on rational use of FDCs for prescribers for cardiovascular morbidities and mortality.
\end{abstract}

Keywords: FDCs, Cardiovascular disorders

\section{INTRODUCTION}

A cardiovascular disease (CVD) is one of the most common cause of mortality and morbidity globally. ${ }^{1}$ The drastic change in the lifestyle of population during $21^{\text {st }}$ century has had an great impact on health especially cardiovascular diseases. Due to implementation of effective prevention and treatment strategies as well as life style modification the developed countries has decreased CVD burden. By contrast, CVD has increased in developing countries and also threatened by poor diet, sedentary life style and obesity, smoking, hypertension, high blood cholesterol and diabetes mellitus. ${ }^{2}$ So our country India is a fertile ground for CVD with major health care burden socially and economically. The common cardiovascular diseases include congestive heart failure, hypertension, cardiac arrhythmia, cerebrovascular accident, coronary artery disease, ischaemic heart disease like angina pectoris and myocardial infarction, aneurysm, atherosclerosis and peripheral vascular diseases. ${ }^{1}$ The prevalence rate of risk factors for CVD has been rapidly rising within India. The annual number of deaths from CVD in India is projected to rise from 2.26 million (1990) to 4.77 million (2020). ${ }^{3}$ As cardiovascular diseases are usually having multiple mechanism in their pathogenesis and frequent presence of co-existing 
disease; clinicians have to prescribe multiple drugs. Multiple drug therapy is associated with poor patient compliance and adherence to treatment so practically it is not successful. Thus, concomitant use of two or more drugs as fixed dose combinations (FDC), whenever suitable, is advised with rationality. Rational Use of Drugs as defined by the world health organization (WHO) depends on making correct diagnosis and prescribing appropriate drugs in adequate doses. ${ }^{4}$ Globally more than $50 \%$ of drugs are prescribed, dispensed or sold inappropriately. ${ }^{5}$ The market is flooded with fixed dose ratio combinations of various drugs, though only few FDCs are rational combinations and are approved by the world health organisation. Irrational prescription of drugs has become common in clinical practice due to lack of knowledge about drugs and also unethical drug promotion. ${ }^{6}$ Irrational use of drugs can lead to high cost of medical treatment increase incidence of adverse drug events and drug misuse. ${ }^{7}$

Hence there is a need to study pattern of prescription of these fixed dose combination drugs and know the rationality behind there usage. Hence we took this study to know pattern of prescription of FDC's in our setting particularly cardiology department.

\section{METHODS}

This was a prospective and observational study conducted at tertiary care hospital Indira Gandhi institute of medical sciences, Sheikhpura, Patna (IGIMS, Patna), Bihar, India. The study protocol was approved from institutional ethics committee of IGIMS, Patna, Bihar, India.

A total of 210 prescriptions of adult patients were collected from cardiology outpatient department (OPD) on daily basis for a period of 6 months from August 2014 to January 2015.

\section{Inclusion criteria}

- Patients of above 20 years and below 90 years of age

- Patients of both sexes

- Patients having cardiovascular disorders

- Patients attending cardiology outpatient department (OPD) on daily basis

- $\quad$ Prescription having FDCs.

\section{Exclusion criteria}

- Patients below 20 years, elderly patients more than 90 years

- Pregnant and lactating women

- Prescription without FDCs

- Indoor patients

- Any serious systemic illness.

\section{Each prescription was studied for}

- Patient's demographic information:- such as patient name, age, sex, address, date of consultation

- Diagnosis and indication

- Prescribed drug: brand name, dose strength, dosage form, frequency, duration and quantity

- Total number of drugs and FDCs in each prescription,

- Composition of the prescribed FDCs,

- Assessment of FDCs whether approved by WHO list of essential medicine or not.

\section{Statistical analysis}

A descriptive analysis of the data was done using Microsoft Excel and results were expressed as percentage.

\section{RESULTS}

Observation of the prescriptions revealed that among the total (210) prescriptions of cardiovascular disorders as well as co-existing illness, a total of 1051 drugs were prescribed. Of this, $260(24.73 \%)$ were FDCs and 791 $(75.26 \%)$ were single dose formulation (Figure 1). Out of the 210 patients, $92(43.80 \%)$ received FDCs (Figure 2).

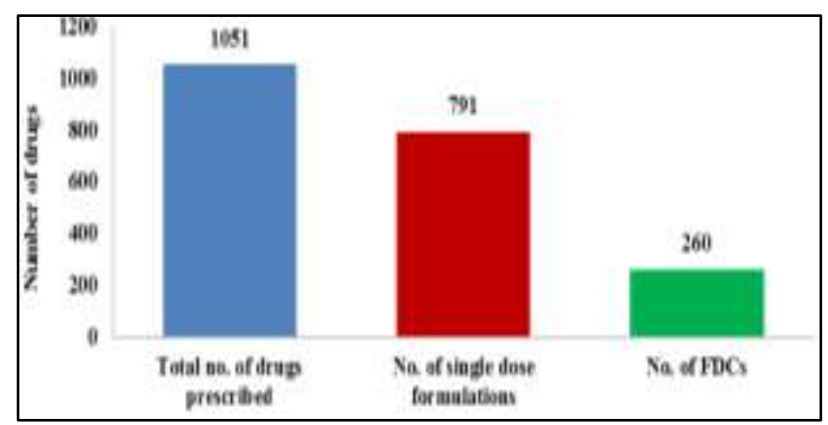

Figure 1: Single dose formulation.

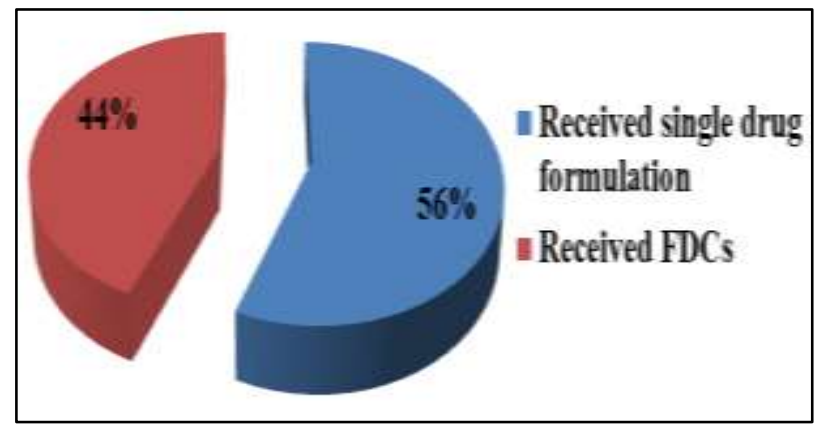

Figure 2: Distribution of Single drug and FDCs in total prescriptions $(n=210)$

Each patient was prescribed with more than 3 medicines. Poly-pharmacy was clearly evidenced in most of the prescriptions. 


\section{Parameters evaluated for prescribing pattern}

Age in years

The age of the patients ranged from 21-80 years. The number of patients who received FDCs was highest in the age group 51-60 years followed by the group 61-70 years of age (Table 1$)$.

Table 1: Age wise distribution of number of patients who received FDCs (92).

\begin{tabular}{|llc|}
\hline Age in years & Number of patients & Percentage $(\%)$ \\
\hline $21-30$ & 1 & 1.08 \\
\hline $31-40$ & 5 & 5.43 \\
\hline $41-50$ & 22 & 23.91 \\
\hline $51-60$ & 28 & 30.43 \\
\hline $61-70$ & 25 & 27.17 \\
\hline $71-80$ & 7 & 7.60 \\
\hline $81-90$ & 4 & 4.34 \\
\hline
\end{tabular}

Sex

Total 210 OPD prescriptions were analysed, $126(60 \%)$ prescriptions were for male category and the remaining 84 (40\%) for female (Figure 3).

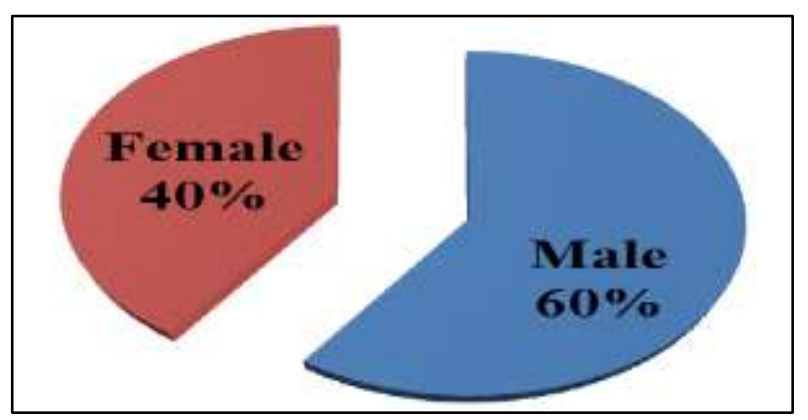

Figure 3: Sex Distribution.

Table 2: Prescribing pattern of FDCs for CVD (N=260).

\begin{tabular}{|lllll|}
\hline Cardiovascular FDCs & Brand names & Strength (mg) & No. of prescriptions & Percentage \\
\hline Aspirin+atorvastatin & Ecospirin-AV & $75+10$ & 42 & 16.15 \\
\hline Telmisartan+hydrochlorothiazide & Telma-H & $40+12.5$ & 33 & 11.69 \\
\hline Furosemide+spironolactone & Lasilactone & $20+50$ & 28 & 10.76 \\
\hline Metoprolol+amlodipine & Metolar-AM & $25+5$ & 24 & 9.23 \\
\hline Ramipril+hydrochlorothiazide & Hopace-H & $2.5+12.5$ & 22 & 8.46 \\
\hline Losartan+hydrochlorothiazide & Losar-H & $50+12.5$ & 21 & 5.07 \\
\hline Amlodipine +losartan & Amlozaar & $5+50$ & 15 & 4.76 \\
\hline Amiloride+furosemide & Amifru & $5+40$ & 12 & 4.61 \\
\hline Clopidogrel+aspirin & Clopitab-A & $75+75$ & 12 & 4.61 \\
\hline Nebivolol+hydrochlorothiazide & Nebistar-H & $5+12.5$ & 11 & 4.23 \\
\hline s-Amlodipine+telmisartan & Espin-TM & $2.5+40$ & 11 & 4.23 \\
\hline Bisoprolol+amlodipine & Zabesta-AM & $5+5$ & 10 & 3.84 \\
\hline Nebivolol+s-Amlodipine & Nebistar-SA & $5+2.5$ & 10 & 3.84 \\
\hline $\begin{array}{l}\text { Losartan+amlodipine+hydrochlorot } \\
\text { hiazide }\end{array}$ & Amlozaar-H & $50+5+12.5$ & 5 & 1.92 \\
\hline Clopidogrel+atorvastatin & Clopitorva & $75+10$ & 4 & 1.53 \\
\hline & & Total & 260 & 100 \\
\hline
\end{tabular}

\section{Common diseases in patients who were prescribed FDCs}

The cardiovascular disorders included congestive cardiac failure (heart failure), cardiomyopathy, cardiac arrhythmias, valvular heart disease, stroke, coronary artery disease, hypertension, ischemic heart disease like myocardial infarction and angina pectoris. The common comorbidities were COPD, bronchial asthma and diabetes mellitus.

Dosage form
All the cardiovascular FDCs prescribed were in oral solid dosage forms.

\section{Duration of therapy}

Among the 210 prescriptions analysed 25 were prescribed up to 7 days, 85 prescriptions for another 15 days and remaining 100 prescriptions for nearly a month.

\section{Therapeutic classification of prescribed FDCs}


A total of 15 types of drug combinations were used in FDCs, majority of FDCs $12(80 \%)$ were belongs to antihypertensive drugs. Two (13.33\%) of them belongs to antiplatelet and hypolipidemic. One (6.67\%) is antiplatelet agent. In all the FDCs, individual components had different pharmacodynamics. So the synergistic action of drug was enhanced. Therapeutic classification was taken from standard pharmacology and therapeutics books (Figure 4).

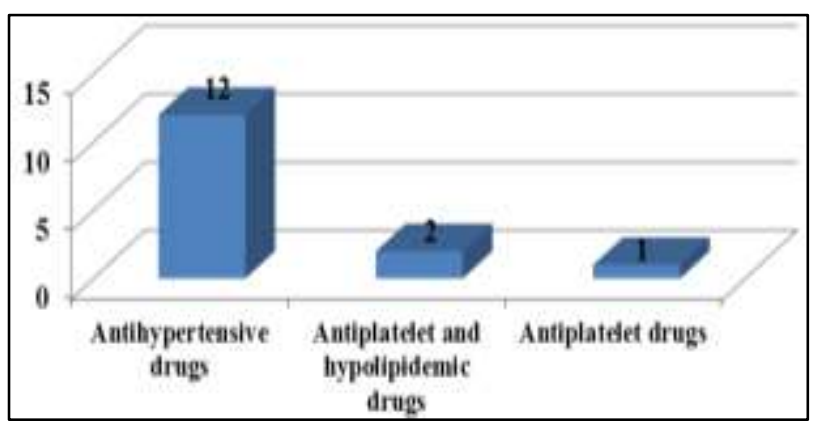

Figure 4: Therapeutic classification of prescribed FDCs.

\section{Assessment of cost of individual drugs and their FDCs}

Analysis of total cost of individual drugs and their FDCs were calculated and compared with the help of CIMS and Drug Today. In 15 different FDCs only 4 (26.67\%) have higher cost than their total cost of individual component, rest $80 \%$ FDCs were found to be more cost effective compared to total cost of individual component .But cost may vary among different brands of pharmaceutical companies prescribed by cardiologist. Sum of cost of individual drugs are more than their FDCs as shown in (Figure 5).

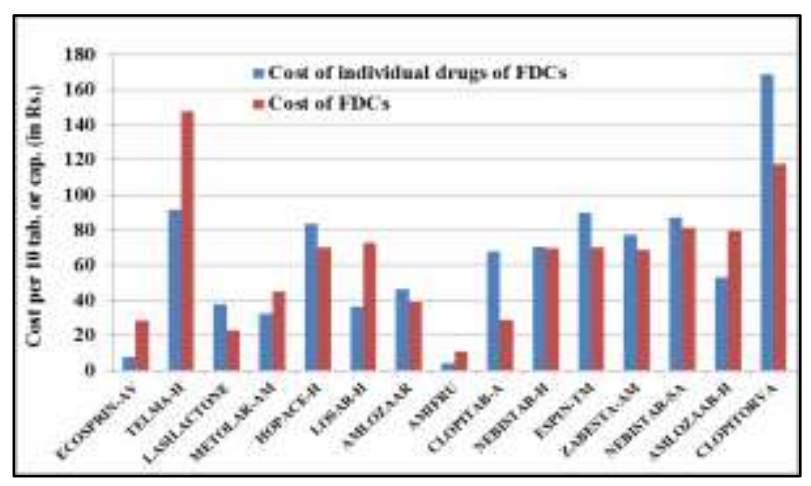

Figure 5: Cardiovascular FDCs.

\section{Determination of rationality}

\section{Number of FDCs prescribed with right indication}

A total of 210 patient prescriptions were analysed which contained 260 FDCs out of which 221 (85\%) FDCs were given for the right indication. Remaining 39 (15\%) FDCs were prescribed without indication.

\section{Number of FDCs prescribed with right dose}

Out of 260 FDCs, $244(93.85 \%)$ FDCs were prescribed with the right dose and remaining $16(6.15 \%)$ FDCs were prescribed with inappropriate dose among 210 analysed prescriptions.

\section{Rationality of FDCs according WHO list}

The rationality of cardiovascular drugs has been determined by referring $19^{\text {th }}$ WHO model list of essential medicines and national list of essential medicines (NLEM).

The results revealed that most of the prescribed single dose drugs are in accordance with the essential model list but the fixed dose combinations prescribed are not included in the list but used commonly.

\section{DISCUSSION}

In 2000, Wald and Law first proposed the use of fixeddose combination therapy for CVD prevention in the form of polypill. ${ }^{8}$ FDCs have many potential advantages like improved patient compliance and adherence to treatment as well as more efficacy, minimal side effects and low cost of therapy. A meta-analysis by Bangalore et al showed that FDCs improve medication compliance.' Increased adherence to treatment among patients treated with FDCs versus separate antihypertensive agents was shown by Gerbino et al. ${ }^{10}$ Another study also supports the use of FDCs in treatment of CVD, combination pharmacotherapy at low doses is likely to be more efficacious than high dose with a monotherapy. ${ }^{11}$ FDCs treat different ailments in the same patient at the same time and with one pill.

It was perceived in our study that most of $(75.26 \%)$ the drugs prescribed were of single dose formulations and $24.73 \%$ were of FDCs.

In our study, out of 260 cardiovascular FDC prescriptions, most of the FDCs belong to the antihypertensive group (80\%) which was found to be similar to that of study conducted by Young et al. ${ }^{12}$

In the present study aspirin+atorvastatin (16.92\%) followed by telmisartan+hydrochlorthiazide (11.53\%), furosemide+spironolactone $(10.76 \%)$ were most commonly prescribed cardio vascular FDCs. This combination reflects the high prevalence of ischemic heart disease and hypertension among study population.

A similar study by Vijayakumar et al reported that amlodipine+atenolol was the most commonly prescribed combination (12.8\%), followed by aspirin+clopidogrel $(11.1 \%)$, and telmisartan+hydrochlorothiazide $(9.8 \%) .{ }^{13}$

Analysed prescriptions of our study shows that more than 3 medicines were prescribed for each patients. The 
average number of drug per prescription should be 2 by WHO recommendation. ${ }^{14}$ So poly-pharmacy was clearly evidenced in our study which may lead to poor patient compliance and treatment adherence. In Rio de Janeiro, Brazil it was found that a mean of 3.7 products per person was used by elderly retirees 60 years of age and older. ${ }^{15}$

The results shows that FDCs prescribed were more in the geriatric age group of 51-60 years which could be because of the multiple mechanisms in pathogenesis of CVD which requires multiple drugs for successful treatment. FDCs is a good alternative, however only a handful of FDCs are rational and justified, while far too many are produced and vigorously promoted by the pharmaceutical industry. In fact, the latest WHO essential medicines list incorporates only 23 FDCs, and the NLEM (2011) of India has only 12 FDCs. ${ }^{16}$

\section{CONCLUSION}

The current study reveals that many FDCs are prescribed by cardiologists. Many of these FDCs are not found where as individual component of FDCs are found in $19^{\text {th }}$ WHO model list of essential medicine (2015). Multifactorial aetiology of CVD requires multiple drugs for treatment justified the use of FDCs to increase treatment adherence as well as improve medication compliance by reducing pill burden of patient. A review and rationalization may be required into the FDCs in essential drug list in view of the many FDCs prescribed for cardiovascular diseases. Further studies are needed in order to validate the efficacy and safety of FDCs which is not included in the current study.

\section{ACKNOWLEDGEMENTS}

The authors thankful to Dr. B.P. Singh, professor and head, department of cardiology, IGIMS, Patna and all other investigators for their support and invaluable help in the completion of this project.

\section{Funding: No funding sources}

Conflict of interest: None declared

Ethical approval: The study was approved by the Institutional Ethics Committee

\section{REFERENCES}

1. World health statistics 2009. Table 2: cause-specific mortality and morbidity. Available at http://www.who.int/whosis/whostat/EN_WHS09_ Table2.pdf. Accessed 2 July 2010.

2. Ara S. A literature review of cardiovascular disease management programs in managed care populations. J Manag Care Pharm. 2004;10:326-44.

3. Ford EA, Ajani UA, Croft JB, Critchley, JaLabarthe DR, KottkeTE, et al. Explaining the decline in coronary mortalityin the United States between 1980 and 2000. N Engl J Med. 2007;356:2388-98.

4. WHO, Model list of essential medicines $19^{\text {th }}$ list. Available

http://www.who.int/medicines/publications/essential medicines/en/.

5. Mohanty BK, Aswini M, Hasamnis AA. Prescription pattern in the department of medicine of a tertiary care hospital in South India. J Clin Diagnostic Res. 2010;4:2047-51.

6. Bhansali NB, Gosai TR, Dholaria NK. Drug utilization study in post-operative patients in surgical ward of a tertiary hospital attached with medical college. Der Pharmacia Lettre. 2013;5(1):251-7.

7. Ranjeeta K, Idris MZ, Vidya B, Anish K, Monika A, Shivendra KS. Assessment of prescription pattern at the public health facilities of Lucknow district. Indian J Pharmacol. 2008;40:243-7.

8. Wald NJ, Law MR. A strategy to reduce cardiovascular disease by more than $80 \%$. BMJ. 2003;326(7404):1419.

9. Bangalore S, Kamalakkannan G, Parkar S, Messerli FH. Fixed-dose combinations improve medication compliance: a meta-analysis. Am J Med. 2007;120(8):713-9.

10. Gerbino PP, Shoheiber O. Adherence patterns among patients treated with fixed-dose combination versus separate antihypertensive agents. Am J Health Syst Pharm. 2007;64(12):1279-83.

11. Combination pharmacotherapy and public health research working group. Combination pharmacotherapy for cardiovascular disease. Ann Intern Med. 2005;143(8):593-9.

12. Young CL, Dias VC, Stangier J. Multiple-dose pharmacokinetics of telmisartan and of hydrochlorothiazide following concurrent administration in healthy subjects. J Clin Pharmacol. 2000;40:1323-30.

13. Vijayakumar TM, Poovi G, Swaroop TVSS, Thirumurugan G, Dhanaraju MD. Prescribing pattern of fixed dose combinations: focus on cardiovascular drugs in outpatient department of private hospitals. Journal of pharmacology and toxicology., 2010;5(5)215-21.

14. Sharif SI, Shaqra M, Hajjar H. Shamout L. Wess. patterns of drug prescribing in a hospital in dubai, United Arab Emirates. Libyan J Med. 2007;3.

15. Rozenfeld S, Fonseca MJ, Acurcio FA. Drug utilization and poly pharmacy among the elderly: a survey in Rio de Janeiro City, Brazil. Rev Panam Salud Publica. 2008;23:34-43.

16. KD Tripathi. Aspects of pharmacotherapy, clinical pharmacology and drug development. Essentials of medical pharmacology. $7^{\text {th }}$ ed. New Delhi: Jaypee; 2013:61-62.

Cite this article as: Manjhi PK, Mohan L, Kumar M, Dikshit H, Singh BP, Pathak AK, et al. Prescription pattern of fixed dose drug combination in cardiology department in a tertiary care hospital. Int J Basic Clin Pharmacol 2016;5:951-5. 